UCRL-TR-210983

\title{
Local-Scale Atmospheric Reactive Flow Simulations
}

Charles K. Westbrook, Robert L. Lee

March 29, 2005 


\section{Disclaimer}

This document was prepared as an account of work sponsored by an agency of the United States Government. Neither the United States Government nor the University of California nor any of their employees, makes any warranty, express or implied, or assumes any legal liability or responsibility for the accuracy, completeness, or usefulness of any information, apparatus, product, or process disclosed, or represents that its use would not infringe privately owned rights. Reference herein to any specific commercial product, process, or service by trade name, trademark, manufacturer, or otherwise, does not necessarily constitute or imply its endorsement, recommendation, or favoring by the United States Government or the University of California. The views and opinions of authors expressed herein do not necessarily state or reflect those of the United States Government or the University of California, and shall not be used for advertising or product endorsement purposes.

\section{Auspices Statement}

This work was performed under the auspices of the U. S. Department of Energy (DOE) by the University of California, Lawrence Livermore National Laboratory (LLNL) under Contract No. W-7405-Eng-48. The project (02-ERD-027) was funded by the Laboratory Directed Research and Development Program at LLNL. 


\title{
FY04 LDRD Final Report Local-Scale Atmospheric Reactive Flow Simulations LDRD Project Tracking Code: 02-ERD-027 Charles Westbrook, Principal Investigator Robert L. Lee, Co-Principal Investigator
}

\begin{abstract}
A computer model was developed to simulate the spatial and chemical evolution of gaseous and aerosol chemicals released into the atmosphere. The evolution is followed over the range of a few kilometers, in environments including terrain variability, urban features including buildings, and variable winds. Submodels for both gas phase chemicals and the chemical composition of liquid and particulate aerosols are included, and preliminary tests of the model are described.
\end{abstract}

\section{Project Background and Motivation}

During 2001, NAI program managers began to discuss with both CMS and $E \& E$ directorate scientists the feasibility of developing a new computer modeling tool to assist in some of their non-proliferation projects. Two capabilities were requested that complemented each other quite strongly. Both involved having the capability to predict the chemical evolution of toxic and other materials as they are carried about by air flow in the atmosphere, primarily in urban environments. In one application, the material to be followed was to consist of gas phase chemical species, which could be imagined to represent some type of chemical warfare species such as Sarin (GB) or VX. The second application involved following the evolution of bacterial and other spores in the atmosphere, an example of which could be anthrax.

In both classes of applications, the gaseous chemical or spore could reasonbly be expected to be convected and diffuse while being carried along in the air, but they could also be expected to react during flight and be transformed into something more or less toxic or made into something completely harmless. The types of reactions that could affect both gaseous chemicals and spores include photolytic reactions from sunlight, reactions with other chemical species commonly present at some level in the urban environment or by chemical species particularly common in urban environments including ozone, oxides of nitrogen, oxides of sulfur, or others. In addition, water vapor and actual liquid water would be expected to be very reactive with these same types of materials.

The code being requested made this a clear task that involved both the Chemistry and Materials Science and the Energy and Environment Directorates, since it required both chemistry and atmospheric flow models, so a collaborative 
ERD project was defined, to be supported by both directorates. Each would supply the expertise and computational tools they possessed, and each would participate in the production of this new capability.

Atmospheric flow models for these same types of urban and other environments already existed at LLNL and elsewhere; the LLNL code was called FEM3D, which was an acronymn for Finite Element Method, 3 Dimensional model. The spatial range to be simulated in the NAI problems was in the range horizontally of 1 to $10 \mathrm{~km}$, and this was the same range for which FEM3D had been designed. The code was available on the LLNL ASC massively parallel computer platforms and on workstations as well, running in parallel or in serial mode, with a considerable variety of problem generation tools and geometrical complexity easily available. A considerable number of new and compatible physics modules were also available, such as the ability to couple to larger scale atmospheric flows, and the ability to include challenging features such as "rain out" and dry deposition on different types of terrain. Another valuable feature was the enormous amount of time and effort that had been invested in the code to include the effects and geometry of buildings on various shapes and sizes, making the code particularly applicable to urban population centers. Furthermore, an extensive set of graphical tools were being developed to visualize the computed results. All of these features made the FEM3D code an attractive one on which to build a new chemistry capability.

An example of a pre-existing capability in the FEM3D code is shown in Figure 1, in which one picture shows an aerial photograph of a section of downtown Salt Lake City. The box shown on this photo has been used to define a computational domain for the fluid mechanics calculations in the other section of the Figure, sahowing the spatial resolution in the numerical zoning of the problem and the resolution around each building.

The FEM3D code already had the capability to transport both gas phase chemicals and spores through the atmosphere, but the one feature missing in the code was the ability to have either of these components change as they are transported. Due to the great degree of similarity in the required codes, it was decided to satisfy the needs for a gas phase chemistry evolution code and a condensed phase spore evolution code into two new modules to be integrated into the same FEM3D code.

During this planning phase, we realized that such a code and modeling capability could also be useful for other classes of applications. The "spore" function could be generalized to treat a wider variety of reactive particulate materials to be transported through the atmosphere, each with its own defined evolution mechanisms that would depend on the nature of the condensed phase particulate. One important class of particulates of great interest consists of droplets suspended in the atmosphere, with the most obvious example being water droplet aerosols with other chemicals dissolved in the water droplets. There was already a rich literature of aerosol chemistry that was fairly phenomenological in nature, and more theoretical treatments of aerosol chemistry were also beginning to appear in publications. Another related 
application is the problem of soot particle evolution in the atmosphere, soot produced by mobile transportation sources, especially diesel engines, and by stationary sources such as factories and power plants. Each would require a different chemistry treatment to predict their evolution, and each would almost certainly be affected differently by solar radiation, atmospheric chemicals and water vapor, and each would have different transport properties due to variations in density, size, radiation absorption, and others, but if each of these features could be identified, the same particulate or aerosol evolution and transport code could treat any of them.

The same situation applies to the gas phase chemical evolution submodel. While the original problem definition applied only to a small class of toxic chemicals, many other environmental problems could also be addressed, such as chemical spills of propane or chlorine, transport and evolution of gaseous air pollutants such as nitric oxide or ozone or gaseous hydrocarbons. Additional examples of both types of atmospheric transport and reaction were also identified as potential uses of the new code capability.

An example of an atmospheric release of a cloud of particulates is shown in Figure 2, with a plume of smoke coming from a fire in a used tire storage facility in central California. The evolution of the cloud, primarily due to shifting wind currents, is evident. The computed path of the smoke plume is indicated by the red computer marker particles in the figure and appears to follow the unintentional experimental path very well. It would be possible to postulate such a fire at selected locations where flammable materials were stored, and combine that with knowledge of locally prevailing wing patterns, to predict the likely path that the smoke would take in the event of a fire. This could provide input into planning the siting of flammable storage facilities, or in the reverse problem where the dump or storage already existed, this could assist in planning construction sites for housing or other facilities. In this example, it would seem obvious that the people living near the storage facility might have some input to the desirability of that type of material being stored close to their homes. 


\section{Code Development}

The major code development tasks involved adding a time-dependent chemical evolution model for both condensed phase and gaseous phase materials. One major assumption was made that limits the applicability of the model as eventually constituted, which is that the material to be transported and evolved chemically is present in amounts sufficiently small that its chemical reactions would not appreciably influence the atmphosperic air flow. Thus this is not intended to be a "combustion" code, where heat release from the reactions produces the bulk motions of the air in which the chemicals are being transported.

The time-dependent evolution code modules for both aerosols and chemicals were then taken from previously existing codes that had been used for other types and scales of simulations, then modified appropriately for the new version of the FEM3D code, and tested in place for a number of problems. The chemistry solver used was a modified version of SMV Gear II, and the new code was given the name FEM3CHEM. Previous tests of FEM3D for non-reacting cases were repeated with both very slow and very fast gas phase chemistry, both of which were problems that could be treated by the older, non-reactive code. Relatively minor developments were required to deal with convergence and stability problems that appeared when the new capabilities were incorporated into the code. In particular, a new non-negative transport algorithm was developed for the CFD advection scheme to eliminate spurious negative concentrations, which greatly improved the overall robustness of the model when chemical reactions are present. We then adapted the chemistry preprocessing component of the global chemistry transport model IMPACT for use in FEM3CHEM.

The gas phase chemistry code was then tested by inserting reaction mechanisms for relatively simple chemicals into an atmopheric flow problem that had previously been solved without reactions. The problem selected for testing was that of a release of dimethyl ether (DME) in a city under realistic atmospheric flow conditions. This mechanism consists of 26 active chemical species and 12 elementary reactions, which has enough detail and complexity to severely test the code capabilities.

Specifically, past experimental results taken from field tests in Salt Lake City, Utah, for preparation studies prior to the recent Winter Olympic Games had released a completely non-toxic and unreactive chemical and measured its time-dependent transport through the city. A picture of the spatial variation in concentrations in shown in Figure 3a. The results from the original calculations were qualitatively similar to the model calculations, and in the present study, we assumed that the chemical released was now DME, which can react during its release and transport, according to a chemical model [1] we defined and entered into the numerical model. Since DME was not the material released in the actual experiment, the computed results represent only a feasibility demonstration of the capability, but the results are quite reasonable. The spatial distribution of a major intermediate product of DME, namely formaldehyde, which is an 
identifed pollutant species from the EPA list of toxics, is shown in Figure $3 b$. These results show that, due to the different molecular weight of the formaldehyde and DME molecules, and due to the different reactivities of these species, they do not simply scale with each other, reaching maximum concentrations at quite different locations. The formaldehyde appears in several somewhat unexpected locations as well, so the non-reactive calculation would not be very accurate in suggesting places where formaldehyde might be present in significant amounts.

We have carried out similar test calculations for the combined fluid mechanics and chemical evolution submodel for aerosols released into an atmospheric flow. We assumed reaction models that were relevant for aerosol evolution and produced sensible results for which we had no experimental basis for comparison. Again, this represents a feasibility demonstration, but experimental validations, not proposed in the original LDRD plan, were not carried out. We performed simulations for hypothetical releases of both toxic and non-toxic aerosols using non-toxics dimethyl methyl phosphonate, methylphosphonic acid, and methoxyphosphonic acid and for the toxic analog Sarin. Again, these computed simulations were carried out for the Salt Lake City test conditions, and these all provided valuable insights into gaseous and aerosol transport and deposition in urban environments.

\section{Code Validation}

While this feasiblity test is perhaps reassuring, it provides a good example of the limitations of our study. There was no provision or intention of including an experimental component in our LDRD project. This was due in part to the limited funds and the cost of fielding experimental tests. However, it also was due to the overall plan for the development of the code capability. We felt that this code, produced by the request of a specific applications group, should be validated separately by each future set of code "clients" for the specific system that they defined for their problems.

There are two major sources of errors or limitations in the predictive power of a model such as this one, attributable roughly to difficulties with the fluid mechanics and the chemistry. The first is the result of accumulated errors in the fluid mechanics of the problem, which could come from inadequate atmospheric flow resolution in the free stream and around obstacles such as buildings, lack of accurate input of prevailing winds and other atmospheric flows, rates of diffusion, rates of chemical deposition on surfaces, and other similar features. All of these have been tested quite thoroughly for the nonreactive versions of the code and compared with available experimental results. This of course does not mean that such errors or problems do not exist, just that many of the limitations and strengths of this code have been independently and previously evaluated.

The second set of possible errors deal with limitations with the chemistry model. The chemical evolution modules used in our demonstration tests have 
been developed for two types of reactive flow systems, representing atmospheric chemistry and combustion chemistry. In some cases these regimes overlap; for example, consumption of hydrocarbons and other related species including DME occurs via radical attack from $\mathrm{OH}$ radicals, and the rates of $\mathrm{OH}$ reaction with DME is known independently for atmospheric and combustion literature. These rates overlap well when extrapolated to common temperatures and pressures, lending confidence to their values. Of course, the $\mathrm{OH}$ radical levels are much different in the open air and in a flame, so the overall rates of this reaction are vastly different, but the chemical model can deal effectively and accurately with such questions.

For processes unique to atmospheric conditions, we have taken the chemical rates of those processes from the appropriate sources. Thus the rates of reaction with solar radiation with DME was taken from the atmospheric chemistry reference database, and this was followed as closely as possible for all other species. However, not all such data are particularly well known, and so there are uncertainties due to lack of specific chemical data. One area in which this is especially true is in the rate of reactions of some species with water vapor in the atmosphere, and we have estimated such data on the basis on species where such information has been measured. Our model computations indicate that reactions between aerosols and ambient water vapor/humidity are particularly sensitive in determining the evolution of the chemical aerosol.

A final, particularly important and also uncertain area, regards the effects of liquid water on the rates of reaction of important chemicals. For many of this class of problems, solubility, solvation and other processes control these effects, and we are currently carrying out some fundamental studies to learn how to model them properly in this type of code environment.

With the exception of the interactions with liquid water, we feel that most of the interesting and important processes are being done properly, subject to unknown rate values and physical processes that we have omitted due to lack of knowledge. Further validation of the composite, kinetic and transport model would require specific experimental tests and comparisons with numerically predicted results. Such tests would probably be carried out first in simplified geometries and carefully controlled conditions.

On the other hand, many applications of this composite code would probably not require detailed validation studies, subject to the specific needs of those using the code. For example, using the results shown in Figure 3, these results can identify a number of large scale phenomena such as recirculation zones behind buildings, the degree to which chemical and biological materials could be transported in different directions relative to the mean air flow, and general strategies for encouraging or avoiding material to be transported to specific types of locations. Guidance on where to put a sensor to detect material released into the atmosphere, where to put people or other resources to avoid possible contamination, and many other features that are useful even at the qualitative level are already available with this computational tool in the absence of further validation studies. 
Note for example the recirculation region in the lower right portion of Figure 3, where the plume appears to flow in an upwind direction and impinge on the large side of the large building in the foreground. The atmospheric flow code predicts that the wake of that building in the prevailing wind direction would bring the chemical material up against the overall wind.

The most important purpose of this LDRD project was to build a reliable framework into which a new chemistry or aerosol reaction module could be inserted, and the resulting composite model could be expected to operate in a stable and sensible manner. Since we could not presuppose any particular chemical or aerosol to be of interest, we assumed that the user of the code would either supply that module at the time of their own project or would commission the code builders to develop a reaction module on request, having defined the materials and conditions of interest.

Many such chemistry models already exist, either in simplified or highly detailed form, and the tools for converting that kind of information into a format usable by the present FEM3D code are reasonably well established. Below we will outline the development of several such submodels which we believed to be of particular interest to our potential collaborators. Some of these submodels were in fact developed under the guidance and funding the present LDRD project, and those chemistry submodels were actually published in the chemical kinetics and combustion literature.

The same is not true of the aerosols of interest to this project. Because of their multiphase nature and the complexity of the evolution of aerosols in both the liquid and solid phases, most aerosol evolution models are simple and rather qualitative. In some applications this is entirely appropriate, but in other cases much more is needed to provide predictive model calculations. Therefore, we have devoted a considerable amount of effort to defining some features of aerosol chemistry that we believe will be useful in the future, and these efforts will be described below. 


\section{Chemical submodel development}

The types of applications for the simulation capabilities described above generally involved the dispersal of toxic chemicals, often including chemical and biological weapons, in the form of gaseous species and as aerosols. Literature searches showed that very little or no information was available for this type of material, and this project devoted some basic research to develop reaction and transport data for inclusion in the FEM3D model. Two major subprojects were carried out to accomplish this task

\section{TASK I Gas Phase Kinetic Models for Organophosphorus Chemical Species}

Many chemical warfare agents consist of variations of a class of organophosphorus compounds (OPC). The simplest such form is di-methyl methyl phosphonate (DMMP), which has a basic structure shown in Figure 4. Quite a lot of experimental and kinetic modeling work has been done for this chemical $[2,3]$, which is not particularly toxic and is certainly not a chemical warfare (CW) agent. The chemical structure of another OPC is also shown in Figure 4, di-isopropyl methyl phosphonate (DIMP), in which the side methyl groups in DMMP have been replaced by isopropyl groups. DIMP is used as a pesticide and has a modest toxicity. The CW agent Sarin, also known as GB, is also shown in Figure 4, and in structural terms, Sarin can be seen to be identical to DIMP, except that one of the isopropyl groups has been replaced by the F atom.

Our kinetic modeling work began with previously existing kinetic models [2] for DMMP and another simple OPC, trimethyl phosphate (TMP). Using established kinetic and thermochemical simulation techniques, we developed kinetic models for DIMP $[4,5,7,8]$ and Sarin [6] in this LDRD project. Among the important scientific results of this work was the observation that the ignition and oxidation properties of Sarin and DIMP were extremely similar to each other, with the reactivity being controlled by the isopropyl radical interactions with the $\mathrm{O}$ atom connected to the $\mathrm{P}$ atom in the OPC. The reactivities of TMP and DMMP were also very similar to each other, both being controlled by $\mathrm{H}$ atom abstraction from the basic OPC structures. However, we also found that the TMP/DMMP species had very different chemical behavior than the DIMP/Sarin species, since the two systems were controlled by different elementary reaction pathways.

This result is important because there is considerable value in having a less toxic alternative to Sarin to use in experimental studies of CW agent effects. In the past, the most convenient alternative, or surrogate, has been DMMP, and the kinetic modeling work we carried out has shown the DMMP is not a particularly accurate surrogate for Sarin. However, DIMP would be a useful surrogate because its reactivity is quite similar to that of Sarin. While the Sarin kinetic model or a kinetically reduced version of it could be used in the model to carry out numerical studies of Sarin dispersal, comparable experimental studies would then be carried out using DIMP with little sacrifice in chemical properties. 


\section{Task II Droplet/air Interface Studies}

Many toxic species are most effectively dispersed when dissolved in water droplets and carried about in the air as an aerosol or spray. Water aerosols can be reactive in the atmosphere, but the details of the interaction of such droplets with solar radiation and with gaseous chemical species in the atmosphere have never been accurately understood. In the present project, we directed our attention to try to understand the nature of the interface between such a droplet and the atmosphere, using the tools of ab initio molecular dynamics (MD) and the massivley parallel supercomputers available at LLNL.

We began by using the Car-Parinello (CP) MD techniques to compute the evolution of 216 water molecules in a region in contact with air. All previous computational studies had been limited by computing resources to use far fewer water molecules, and those past studies had not been able to eliminate false effects due to artifical boundary conditions. Our studies, made possible by the enormous computing resources that were employed, showed that the surface of the water droplet appears to be rich in $\mathrm{H}$ atoms, so it appears chemically acidic to approaching chemical species from the atmosphere. Two valuable papers were published as a result of this work $[9,10]$, including one in the particularly prestigious journal Science. Continuing studies are exploring the similar nature of water droplets which contain other dissolved chemicals such as OPC species, and the eventual goal is to understand whether or not $\mathrm{CW}$ and other toxic chemicals such as oxides of nitrogen are more or less reactive in this aerophase form than in conventional gaseous form in the atmosphere. The results of this analysis could have significant impact on developing ways to mitigate the effects of airborne toxic chemicals.

\section{Conclusions}

The goal of this project was to develop a computer model capable of simulating chemically reactive flows in the atmosphere over a local spatial scale of several kilometers. Gaseous chemicals and aerosols with both liquid and solid phases were included in the systems that could be evolved. This goal was accomplished. Reaction models for a number of gaseous and liquid aerosol species were developed and tested in this overall system model. Future users of this model can use or adapt existing chemical submodels or develop them for new systems. References 1, 3 and 5-10 were published in part or entirely as results of this LDRD project. 


\section{References}

1. Zheng, X.L., Lu, T.F., Law, C.K., Westbrook, C.K., and Curran, H.J., "Experimental and computational study of nonpremixed ignition of dimethyl ether in counterflow", Proc. Combust. Inst. 30, 1101-1109 (2004). Lawrence Livermore National Laboratory, Livermore, CA, UCRL-CONF201586, December 2003.

2. MacDonald, M.A., Jayaweera, T.M., Fisher, E.M., and Gouldin, F.C., "Inhibition of nonpremixed flames by phosphorus-containing compounds", Combustion and Flame 116, 166-176 (1999).

3. Korobeinichev, O.,P., Shvartsberg, V.M., Shmakov, A.G., Bolshova, T.A., Jayaweera, T.M., Melius, C.F., Pitz, W.J., and Westbrook, C.K., “Flame Inhibition by Phosphorus-Containing Compounds in Lean and Rich Propane Flames", Proc. Combust. Inst. 30, 2350-2357 (2004). Lawrence Livermore National Laboratory, Livermore, CA, UCRL-CONF-201585, , December 2003.

4. Glaude, P.-A., Curran, H. J., Pitz, W. J., and Westbrook, C. K.

"Kinetic Study of the Combustion of Organophosphorus Compounds", Proc. Combust. Inst. 28: 1749-1756 (2000). Lawrence Livermore National Laboratory, Livermore, CA, UCRL-JC-137100, October, 2000.

5. Jayaweera, T.M., Melius, C.F., Pitz, W.J., Westbrook, C.K., Korobeinichev, O.P., Shvartsberg, V.M., Shmakov, A.G., Rybitskaya, I.V., and Curran, H.J., "Flame inhibition by phosphorus-containing compounds over a range of equivalence ratios", Combustion and Flame 140, 103-115 (2005). Lawrence Livermore National Laboratory, Livermore, CA, UCRL-JRNL206058, August, 2004.

6. Glaude, P.-A., Melius, C., Pitz, W. J., and Westbrook, C. K., “Detailed Chemical Kinetic Reaction Mechanisms for Incineration of Organophosphorus and Fluoro-Organophosphorus Compounds", Proc. Combust. Inst. 29, 2469-2476 (2002). Lawrence Livermore National Laboratory, Livermore, CA, UCRL-JC-146563, August, 2002.

7. Jayaweera, T.M., Melius, C.F., Pitz, W.J., Westbrook, C.K., Korobeinichev, O.P., Shvartsberg, V.M., Shmakov, A.G., Rybitskaya, I.V., and Curran, H.J., "Flame inhibition by phosphorus-containing compounds over a range of equivalence ratios", Western States Section of the Combustion Institute, Davis, CA, March 2004. Lawrence Livermore National Laboratory, Livermore, CA, UCRL-CONF-202992, March, 2004.

8. Jayaweera, T.M., Pitz, W.J., and Westbrook, C.K., "Suppression of Premixed C3H8-Air Flames by Halogenated and Phosphorus-Containing Compounds" Third Joint Meeting of the U.S. Sections of the Combustion Institute, Chicago, IL, March 2003. Lawrence Livermore National Laboratory, Livermore, CA, UCRL-JC-150885, January 2003. 
9. Kuo, I.-F. W., and Mundy, C.J., "An ab Initio Molecular Dynamics Study of the Aqueous Liquid-Vapor Interface", Science 303, 658-660 (2004). Lawrence Livermore National Laboratory, Livermore, CA, UCRL-JRNL154948, November 2003.

10. Kuo, I.-F. W., Mundy, C.J., McGrath, M.J., Siepmann, J.I., VandeVondele, J., Sprik, M., Hutter, J., Chen, B., Klein, M.L., Mohamed, F., Krack, M., and Parrinello, M., "Liquid Water from First Principles: Investigation of Different Sampling Approaches", J. Phys. Chem. B 108, 12990-12998 (2004). Lawrence Livermore National Laboratory, Livermore, CA, UCRLJRNL-204315, May, 2004. 
Figure captions

1. Photograph of a smoke plume from a tire fire in the San Joaquin Valley of California. Red marker particles from a FEM3D simulation are superimposed on the photograph.

2. (left) Aerial photograph of a portion of Salt Lake City, Utah. Selected building are artificially colored for identification.

(right) Numerically generated spatial grid for computation, representing a portion of the region in the left part of the figure. The vertical grid is not shown.

3. (top) Contour plots at a specific time of initial chemical (dimethyl ether, DME) from a hypothetical release in the grid region of Figure 2. (bottom) Contour plots of formaldehyde concentration, an intermediate of DME oxidation, at the same time as the results for DME shown in the top figure.

4. Schematic structural diagrams of organophosphate compounds and the CW agent Sarin. 


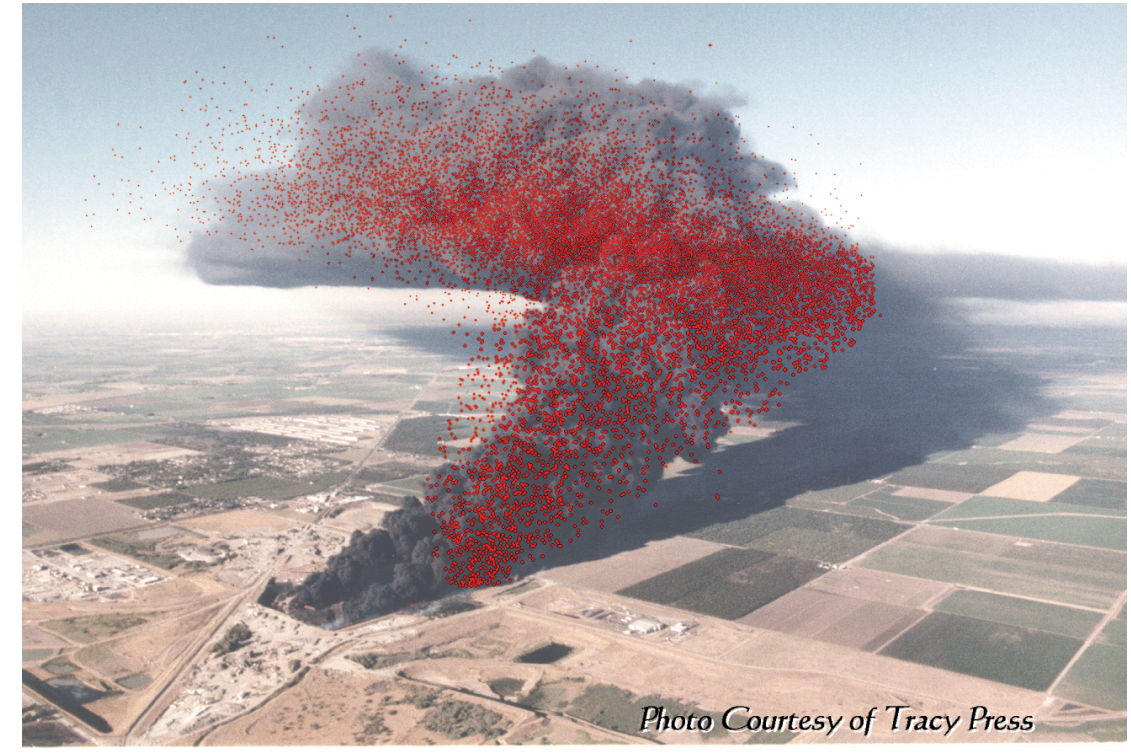

Figure 1 

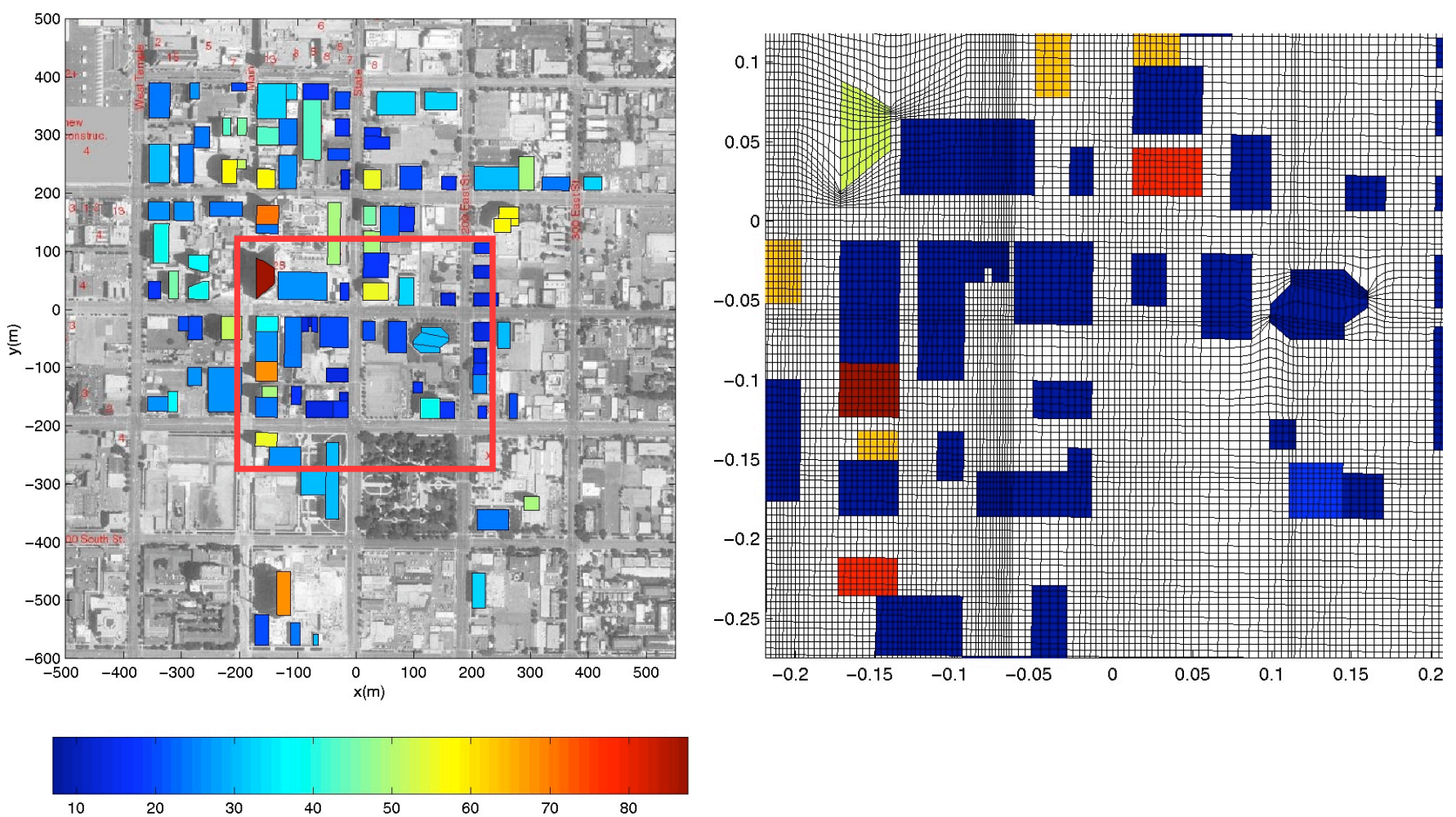

Figure 2 

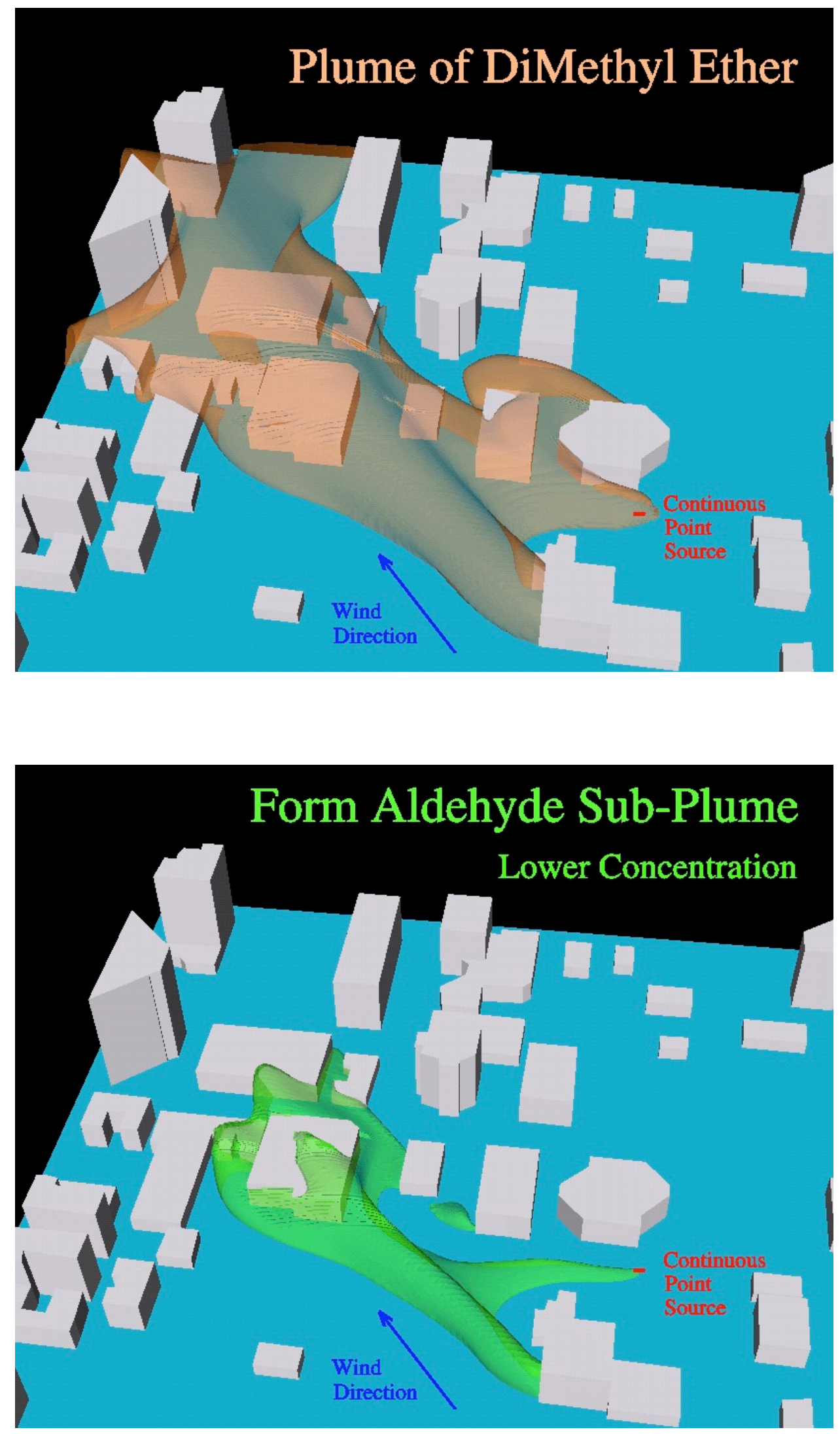

Figure 3 


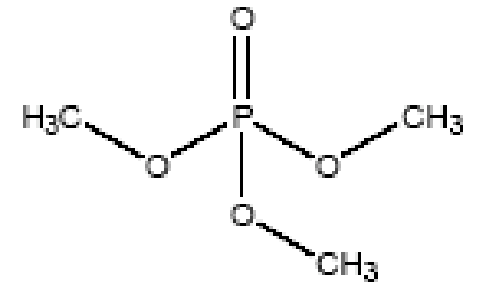

$\underline{\text { TMP }}$

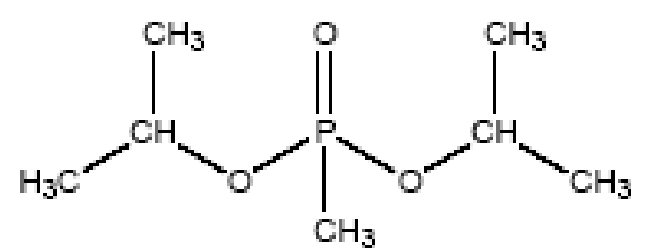

DIMP<smiles>COP(C)(=O)OC</smiles>

DMMP<smiles>CC(C)OP(C)(=O)F</smiles>

$\underline{\text { Sarin (GB) }}$ 